\title{
Experimental Investigation and Verification of Traps affecting the performance of 3C-SiC-on-Si Schottky Barrier Diodes
}

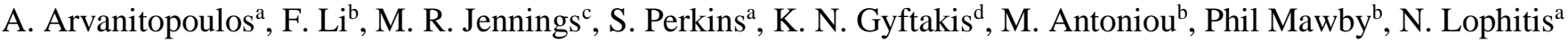 \\ ${ }^{\text {a }}$ Faculty of Engineering, Environment and Computing, Coventry University, Coventry, UK \\ ${ }^{\mathrm{b}}$ School of Engineering, University of Warwick, Coventry, UK \\ ${ }^{c}$ College of Engineering, Swansea University, Swansea, UK \\ ${ }^{d}$ School of Engineering, The University of Edinburgh, Edinburgh, UK \\ email: arvanita@uni.coventry.ac.uk
}

\begin{abstract}
Monolithic integration of wide bandgap (WBG) devices for power integrated circuits is currently of increased interest. The ability of the cubic phase (3C-) of Silicon Carbide (SiC) to grow heteroepitaxially on Silicon $(\mathrm{Si})$ substrates $(3 \mathrm{C}$ $\mathrm{SiC}$-on-Si) is an enabling feature for a cost-effective integration, including the possible integration with $\mathrm{Si}$ devices. The isotropic properties of $3 \mathrm{C}$-SiC and the high thermal conductivity also emphasize its importance. In this paper, the authors evaluated the actual $3 \mathrm{C}$-SiC-on-Si material and established a feasible fabrication methodology. In achieving this, non-freestanding lateral Schottky Barrier Diodes (LSBD) have been fabricated and tested. To gain a deep physical insight of the complex phenomena that take place in this material, an advanced Technology Computer Aided Design (TCAD) model was developed which allowed accurate match of measurements with simulations. The model incorporated the device geometry, an accurate representation of the bulk material properties and complex trapping/de-trapping and tunnelling phenomena which appear to affect the device performance. The observed nonuniformities of the Schottky Barrier Height (SBH) were also successfully modelled through the effect of the interfacial traps. The combination of TCAD with fabrication and measurements enabled the identification of the trap profiles and their impact on the electrical performance of this new material, a necessary step towards device designs that take advantage of its properties.
\end{abstract}

Keywords-Schottky contacts, inhomogeneity, traps, SiC, 3CSiC-on-Si, TCAD

\section{INTRODUCTION}

The demands of modern applications in power electronics make the Silicon- ( $\mathrm{Si}$ ) based power devices lag, mainly due to the moderate critical electric field value of the material [1], [2]. On the other hand, wide bandgap (WBG) semiconductors, like Silicon Carbide (SiC), promise advanced properties [3][5]. The $3 \mathrm{C}-\mathrm{SiC}$, also referred to as $\beta-\mathrm{SiC}$, is the only form of $\mathrm{SiC}$ with a cubic crystal structure, similar to $\mathrm{Si}$ [6]. In consequence, its ability to heteroepitaxially grow on large $\mathrm{Si}$ substrates (3C-SiC-on-Si) meets the low-cost requirement and, concurrently, encourages possible integration with $\mathrm{Si}$ devices [7], [8]. The induced isotropic properties of 3C-SiC due to its cubic structure, along with its refractory nature, the superior electron mobility at high temperatures and the high thermal conductivity also emphasize its importance [9]-[11].

The larger energy band gap of 3C-SiC allows higher doping and thinner layers for power devices with given blocking capabilities. Thus, resulting in much lower specific on-resistance, particularity for unipolar devices, like Schottky Barrier Diodes (SBDs). Nonetheless, these diodes do not reach their full potential due to an observed excessive leakage current [12]. This deteriorates their performance in both forward and reverse bias conditions and hence, currently obstructs them from commercialization. Notably, when the $3 \mathrm{C}-\mathrm{SiC}$ grows on $\mathrm{Si}$, various types of bulk traps are formed in the epitaxial layers [13]-[17], due to lattice and thermal mismatches at the heterointerface [18]. The electrical activity of such extended defects in 3C-SiC-on-Si, like stacking faults (SFs) and micro-twins [19], is a major concern for functional power devices. As a matter of fact, the current flows preferentially through these defects, leading to high leakages and low breakdown voltages. In addition, the deposition of a metal on any semiconductor leads to the formation of supplementary electronic states at the Schottky interface [20]. The presence of such a variety of traps affects the metal / 3C$\mathrm{SiC}$ interfaces and the formation of Schottky barriers in particular [21].

Furthermore, reported inhomogeneous features of the Schottky contact can essentially add to the resulted leakage current before the onset of forward conductivity or while in blocking mode [22]. In the literature there have been various attempts to model such features. In [23]-[25] a simplified approach assumed the contact splitted in two parts, each one characterized by an independent SBH value. This introduced the concept of parallel conduction in which two or more discrete barrier heights are operating in parallel at the Metal / $\mathrm{SiC}$ interfaces [26]-[30]. In a similar manner, in [31]-[33] the SBH values were modelled with a Gaussian distribution resulting in a non-uniform behaviour of the Schottky contact under voltage stress. A very well established model was developed by Tung to describe the inhomogeneity observed in fabricated Schottky contacts and has emerged as the most complete method to incorporate parallel conduction methodology [34], [35]. According to Tung's theory, the inhomogeneous behaviour of the SBH is attributed to potential fluctuations at the Schottky interface, but no assumptions are made on the origins of this observed inhomogeneity [24]. These fluctuations are considered as a localized phenomenon and spatially limited on small portions of the contact, called patches [36]. The Tung's model is applied by determining the values of fitting parameters from a modified Thermionic Emission (TE) equation based on the suggestion that neighbouring patches interact. However, questions have been raised about the physical interpretation of the fitting parameters of this model for diodes that widely deviate from the ideal TE behaviour $(\eta>1.21)$, irrespective of the material system [24], [37]. Such obtained highly unphysical parameters indicate that the source of the significant degree of 
non-ideality in these SBDs is possibly extrinsic in nature, i.e. traps and/or process induced impurities.

It is of interest that both the degradation of the electrical performance and the observed inhomogeneous of a Schottky contact have been linked with the presence of traps in fabricated SBDs [38]-[43]. However, the characterization of these traps' and their overall influence on the barrier height value of 3C-SiC-on-Si SBDs are still mostly unknown and unclear [25].

In this paper, 3C-SiC-on-Si lateral SBDs (LSBDs) were fabricated to perform an experimental investigation of the traps affecting the carrier transport mechanisms. Based on validated 3C-SiC material models [44], [45], an advanced Technology Computer Aided (TCAD) model is developed allowing for the identification of the trap profiles in the diode. These traps profiles include both bulk traps and Schottky interface states. The effect induced to the Schottky Barrier Height (SBH) by these traps was revealed with simulations and fully justifies the high leakage current observed in the fabricated diodes. Furthermore, the proposed model also attributes non-uniformities of the $\mathrm{SBH}$ to additional trap profiles at the Schottky interface. The multiple interfacial trap profiles are then differentiated by distinct spatial distributions on the active area of the contact. Although the simplicity of a LSBD, this structure allows for gaining the insights of the traps' impact on the leakage current. The existence of the traps is correlated with complex trapping/de-trapping and tunnelling phenomena that enhance the recombination/ generation mechanism of carriers, depending on the bias polarity. Thus, the proposed model is further applicable for diodes that exhibit high ideality factor values. To the credit of this work, the modelled influence of the traps is not limited to 3C-SiC-on-Si SBDs but can be also applied for any SiC SBD.

\section{DEVICE FABRICATION}

Circular and rectangular/striped contacts have been fabricated on the $3 \mathrm{C}-\mathrm{SiC}-$ on-Si wafer. The grown $3 \mathrm{C}-\mathrm{SiC}$ layer is $4.28 \mu \mathrm{m}$ thick and of $5 \times 10^{16} \mathrm{~cm}^{-3}$ Nitrogen $(\mathrm{N})$ doped. The Si substrate utilized is Boron (B) doped and features $\mathrm{p}$ type conductivity characteristics with a resistivity value of 5$10 \mathrm{Ohm} \cdot \mathrm{cm}$. Nitrogen with doping around $5 \times 10^{20} \mathrm{~cm}^{-3}$ was implanted in selective regions and activated at $1350^{\circ} \mathrm{C}$ for 2 hours. Ohmic contacts were obtained on these implanted regions by depositing a Ti30nm/Ni100nm bilayer and annealed at $1000^{\circ} \mathrm{C}$ for $1 \mathrm{~min}$. The Schottky contacts were formed by evaporating $200 \mathrm{~nm}$ of Platinum $(\mathrm{Pt})$ and no further treatment was applied.

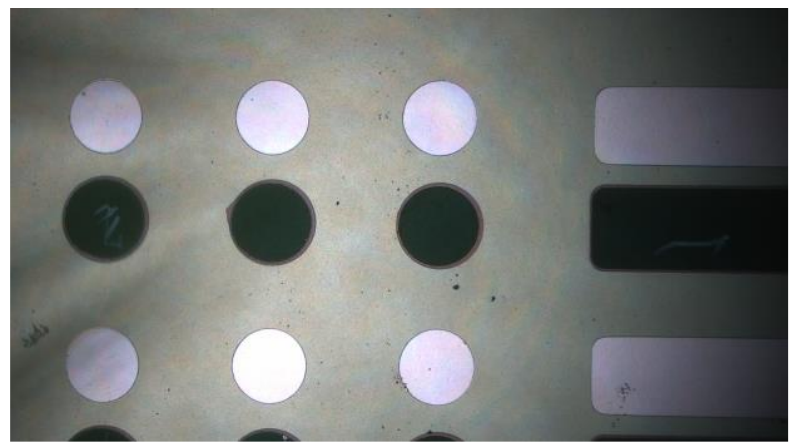

Fig. 1 Both circular and striped LSBDs were fabricated on 3C-SiC-on-Si samples.

A sample of the fabricated LSBDs on 3C$\mathrm{SiC}(100) / \mathrm{Si}(100)$ is shown in Fig. 1. The dark coloured contacts are the ohmic contacts, whilst the brightest correspond to the Pt-Schottky ones. To obtain diode variants, two design variables were introduced in the design split. The diameter and/or the width accounted as the first variable for the circular and the rectangular contacts correspondingly. The circular shaped LSBDs featured $\emptyset 100 \mu \mathrm{m}$ for the smaller contacts and $\emptyset 150 \mu \mathrm{m}$ for the larger ones. Accordingly, small rectangular contacts were of $100 \mu \mathrm{m}$ width, whereas the large ones were of $150 \mu \mathrm{m}$ width. The lateral separation between the ohmic and Schottky contacts was the second design variable with values ranging 30,35 and $40 \mu \mathrm{m}$.

\section{DeVice Modelling Methodology}

The B1505A equipment [46] was utilized for the characterization process. Thereafter, the ideality factor $(\eta)$ was calculated from the I-V measurements for each LSBD. Most devices were found to feature large $\eta$ values. In addition, the ideality factor of a diode was not constant rather it demonstrated a varying value with the applied forward bias. Based on their lowest $\eta$ value exhibited, the small rectangular diodes were chosen for TCAD modelling and analysis through simulations.
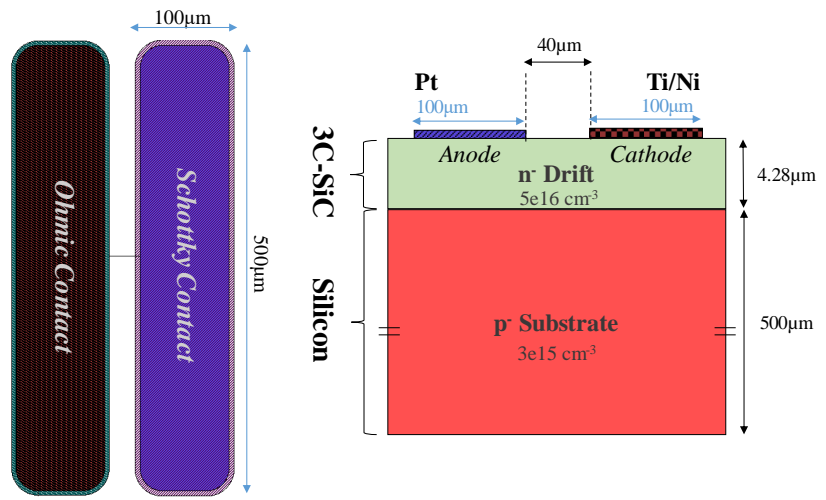

Fig. 2 The top view (left side) and cross-section (right side) of the 3C$\mathrm{SiC}(100) / \mathrm{Si}(100) \mathrm{LSBD}$ characterised and modelled.

The cross-section view of the investigated as-deposited $\mathrm{Pt} / 3 \mathrm{C}-\mathrm{SiC}$-on-Si LSBD is illustrated in Fig. 2. The TCAD simulated striped structure is based on state-of-the-art 3C-SiC material physical models [44]. The 3C-SiC material layer of $4.28 \mu \mathrm{m}$ thickness and $5 \times 10^{15} \mathrm{~cm}^{-3}$ Nitrogen $(\mathrm{N})$ doped corresponds to the drift region. Hence, electrons act as the majority carriers. The Schottky metal is Platinum with a workfunction of $q \Phi_{M}=5.65 \mathrm{eV}$ [47]. The Schottky and ohmic contacts are laterally separated by $40 \mu \mathrm{m}$. The $\mathrm{Si}$ substrate is considered lowly doped and of opposite conductivity type compared to the drift, according to the wafer specifications. An area factor has been utilized to accurately represent the diode in the third dimension. Towards accurate simulations, additional to the Thermionic Emission (TE), the barrier lowering due to image force and the barrier tunnelling physical mechanisms are also considered.

\section{VERIFICATION OF TRAPS AFFECTING THE PERFORMANCE OF THE FABRICATED 3C-SIC-ON-SI LSBD}

Focusing on the on-state of the I-V characteristics of the fabricated diode, simulations were performed with the modelled structure in Fig. 2. Matching the simulations with the experiments, within this bias region, allowed for the identification of existing bulk traps in the drift of the investigated 3C-SiC-on-Si LSBD. The determined properties of these trap levels are presented in Table I. Their verified 
behaviour as deep acceptors resembles the intrinsic Silicon Vacancies $\left(V_{S i}\right)$ defects [48]. Such defects are common in the 3C-SiC-on-Si originating from the out-diffusion of the $\mathrm{Si}$ atoms to form the $\mathrm{SiC}$ layer [7]. The activation energy of these bulk traps, as given in Table I, indicates a high probability of their being occupied with majority carriers even at zero bias, therefore, an effective Fermi level $\left(E_{F}^{e f f}\right)$ is considered for $3 \mathrm{C}-\mathrm{SiC}$. This is illustrated in the band diagram in Fig. 3. Notably, in thermal equilibrium the relative position of the $E_{F}^{\text {eff }}$ at the Schottky interface $\left(\left.E_{F}^{e f f}\right|_{I T}\right)$ also changes to a lower energy.

Table I. Identified trap profiles in the fabricated and investigated 3C-

\begin{tabular}{|c|c|c|c|c|}
\hline \multirow{2}{*}{\multicolumn{2}{|c|}{ Trap specification }} & \multirow{2}{*}{$\begin{array}{c}\text { Type / } \\
\text { Concentration }\end{array}$} & \multicolumn{2}{|c|}{ Distributions } \\
\hline & & & Energetic & Spatial \\
\hline$\stackrel{4}{5}$ & $\begin{array}{c}\text { Deep } \\
\text { levels due } \\
\text { to } 3 C \text { - } \\
\text { SiC/Si } \\
\text { hetero- } \\
\text { interface } \\
\text { to model } \\
\text { the } \\
V_{S i} \text { defects }\end{array}$ & $\begin{array}{c}4.85 \times 10^{16} \mathrm{~cm}^{-3} \\
\text { of } \\
\text { Acceptors }\end{array}$ & $\begin{array}{l}\text { Single Level } \\
\text { Activation } \\
\text { Energy } \\
E^{A}=0.5 \mathrm{eV} \\
\left(\text { from } E_{V}^{3 C}\right)\end{array}$ & $\begin{array}{l}\text { Uniform } \\
\text { covering } \\
\text { all the } \\
\text { drift } 3 \mathrm{C} \text { - } \\
\mathrm{SiC} \\
\text { layer }\end{array}$ \\
\hline$\sum_{0}^{\infty}$ & $\begin{array}{c}\text { Schottky } \\
\text { contact } \\
\text { interfacial } \\
\text { defects }\end{array}$ & $\begin{array}{c}8 \times 10^{18} \mathrm{~cm}^{-3} \\
\text { of } \\
\text { Donor-like } \\
\text { states }\end{array}$ & $\begin{array}{c}\text { Uniform Band } \\
E_{\text {Mid }}=0.6 \mathrm{eV} \\
E_{\text {Sig }}=1.0 \mathrm{eV} \\
\left(\text { from } E_{C}^{3 C}\right)\end{array}$ & $\begin{array}{c}\text { Uniform } \\
\text { along all } \\
\text { the }\end{array}$ \\
\hline 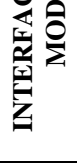 & $\begin{array}{l}\text { in a } \\
\text { specific } \\
\text { effect on } \\
\text { the barrier } \\
\text { height }\end{array}$ & $\begin{array}{l}2 \times 10^{18} \mathrm{~cm}^{-3} \\
\text { of } \\
\text { Acceptor-like } \\
\text { states }\end{array}$ & $\begin{array}{c}\text { Uniform Band } \\
E_{\text {Mid }}=0.2 \mathrm{eV} \\
E_{\text {Sig }}=0.4 \mathrm{eV} \\
\left(\text { from } E_{C}^{3 C}\right)\end{array}$ & $\begin{array}{l}\text { in the } \\
\text { lateral } \\
\text { direction }\end{array}$ \\
\hline
\end{tabular}

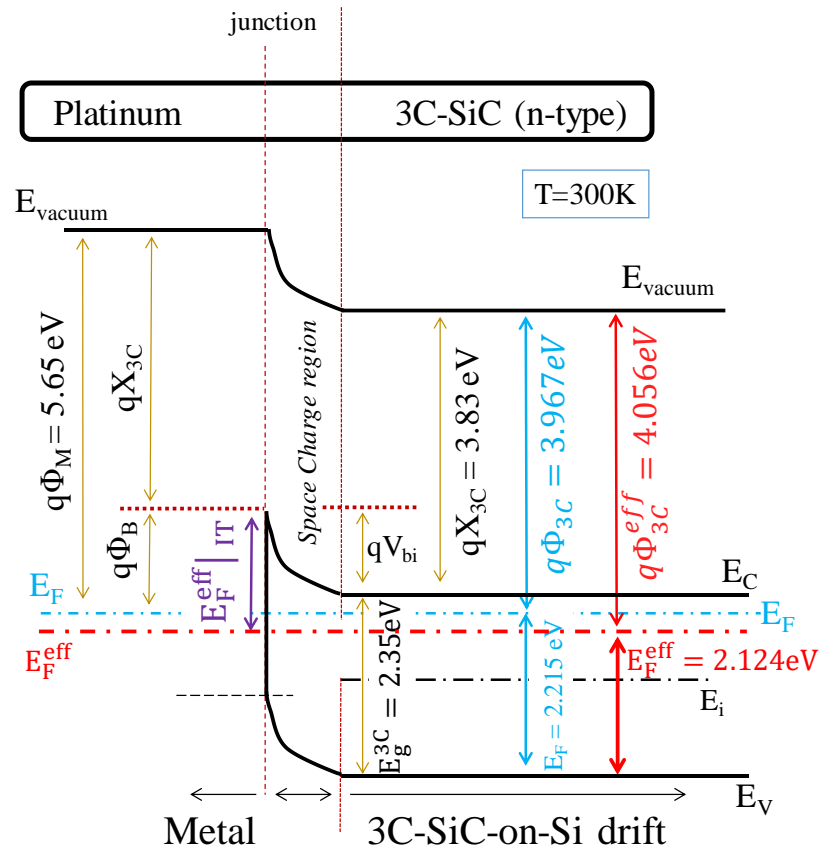

Fig. 3 In zero bias and $\mathrm{T}=300 \mathrm{~K}$ the deep bulk acceptor levels, resembling $V_{S i}$, are highly likely to capture majority carriers, and thus, alter the energy of the Fermi level from an initial $E_{F}$ into $E_{F}^{e f f}$ in the band diagram.

\section{A. Initial consideration with a spatially uniform effect of the interfacial traps over all the Schottky contact}

Although this specific fabricated LSBD resulted in the lowest ideality factor values, these values of $\eta \approx 3$ are still larger than unity, as shown in Fig. 4, indicating a poor
Schottky contact. Furthermore, the sub-threshold region of the forward $\log (\mathrm{I})-\mathrm{V}$ is directly linked to the quality of the semiconductor material [49]. This can be interpreted with the existence of traps both at the Schottky interface and the drift. The observed variations of the value of $\eta$ with the applied voltage signifies that the effect of these traps depends on the bias level and, thus, the Fermi level $\left(E_{F}\right)$ position [50].

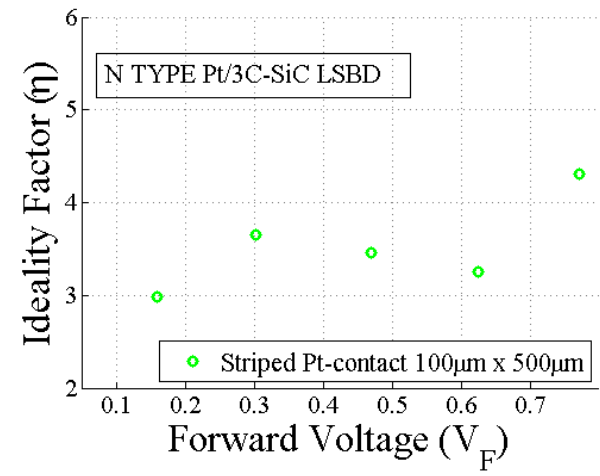

Fig. 4 The calculated $\eta$ values with a step of 60 measurements of the applied forward voltage for the fabricated and characterized $3 \mathrm{C}-\mathrm{SiC}$-on-Si LSBD (modelled in Fig. 2).

In order to replicate the measurements with simulations, an advanced TCAD model is proposed, which suggests the inclusion of further interfacial traps in the simulations and their sub-sequent effect on the carrier transport mechanisms. The properties of these interfacial (Model A) traps were also determined through FEM and are presented in Table I. Particularly, the interfacial Model A consists of both a distribution of donor-like states and a distribution of acceptorlike states. An illustration of how these states are energetically distributed at the interface between $\mathrm{Pt}$ and $3 \mathrm{C}-\mathrm{SiC}-\mathrm{On}-\mathrm{Si}$ is shown in Fig. 5. Thereafter, the calculated relative position of the $E_{F}^{e f f}$ at the interface decides which of these states are ionized, and, thus, highly likely to become occupied by a carrier.

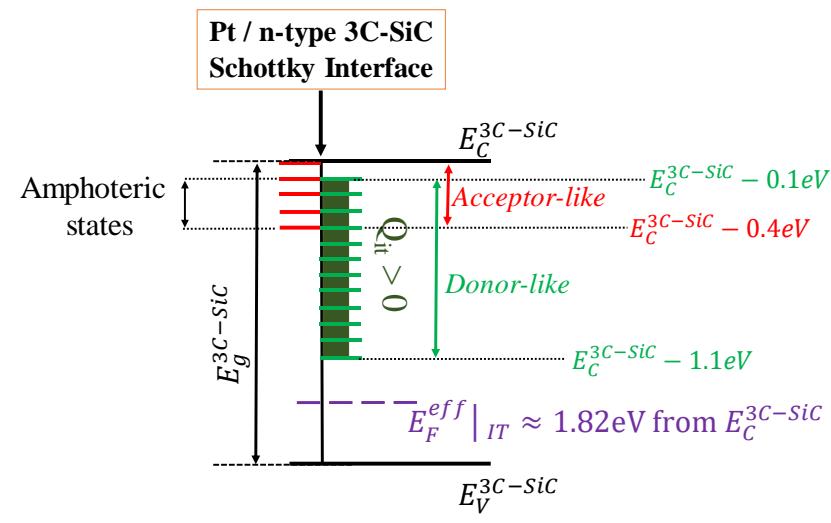

Fig. 5 The energetic distributions of the identified traps' model A at the Schottky interface. The position of the $\left.E_{F}^{e f f}\right|_{I T}$ decides which interfacial states are highly likely to be occupied depending on their type.

It is clear, from Fig. 6, that the TE of majority carriers cannot accurately predict the shape of the $\log (\mathrm{I})-\mathrm{V}$ when traps exist at the Schottky interface. This observation is in agreement with the large calculated ideality factor values of the investigated LSBD. The elevated forward leakage current can be attributed to the presence of the interfacial traps in Table I. In traps' Model A, the donor-like states at the Schottky interface feature high concentration. These states, at the interface, energetically located above the $E_{F}$ are able to 
capture a hole emulating fixed positive charge. The electric field originating from the space charge region is now further enhanced. Hence, although in the early forward bias region, at the interface region the applied electric field increases stimulating the effect from phenomena mainly occurring in reverse bias. In particular, the barrier lowering allows for more majority carriers to cross the barrier through Thermionic Field Emission (TFE). Moreover, due to the high population of occupied donor-like states, the triangular-shaped barrier becomes thin enough to make the Field Emission (FE) a great contributor to the sub-threshold current.

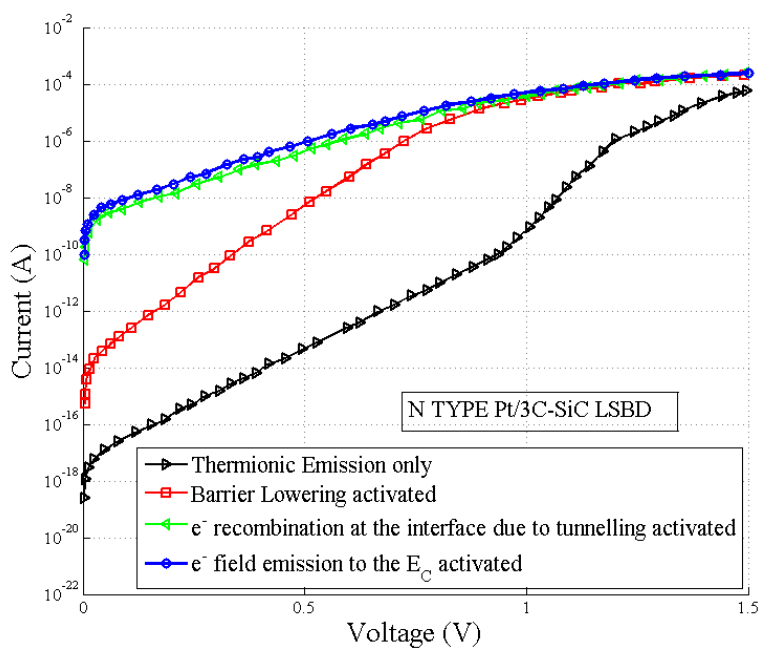

Fig. 6 The identified traps in Table I create the conditions for enhanced carrier transport mechanisms and, thus, a large sub-threshold current that the TE alone is not able to describe accurately. Such mechanisms include barrier lowering, recombination at the interface and direct tunnelling.

Part of the electrons that field emit through the barrier contribute to the current of the investigated 3C-SiC-on-Si SBD by completing a transition to the conduction band $\left(E_{C}\right)$ of the Metal. In addition, recombination of carriers also occurs at the interface. For recombination to happen both types of carriers need to be apparent. The metal system is assumed to be a pool of electrons and holes, thus contributing with holes. Thus, part of the majority electrons tunnel through the Schottky heterointerface, between $\mathrm{Pt}$ and $3 \mathrm{C}-\mathrm{SiC}$, recombine with holes at the valence band $\left(E_{V}\right)$ of the metal. Both the direct FE and recombination at the Schottky interface due to tunnelling have a strong impact on the ideality factor, deviating its value towards values greater than unity.

The amphoteric interfacial states highlight a set of energy levels that correspond to traps which can either behave as donors or acceptors. As long as the external bias level indicates the $E_{F}$ at the Schottky interface is below these amphoteric states they are able to capture holes. With the increasing forward bias, the energy bands move upwards and these states will eventually drop below the $\mathrm{E}_{\mathrm{F}}$. In this case, they act as acceptors and are able to capture electrons. The latter, decreases the number of the majority carriers that can contribute to the sub-threshold current, or locally increases the resistance. Hence, the energetic distribution of the acceptorlike states in Model A, in Table I, has been specified so in order to balance the strong effect from the donor-like states of Model A. Interestingly, the acceptor-like states demonstrated a negligible contribution to the Trap-Assisted Tunnelling (TAT) recombination. This is attributed to the properties of the donor-like distribution of Model A, which energetically expands up to the $\mathrm{E}_{\mathrm{C}}$. The resultant strong band bending at the
Schottky interface, therefore, encourages the direct FE rather than the indirect TAT transition for the electrons.

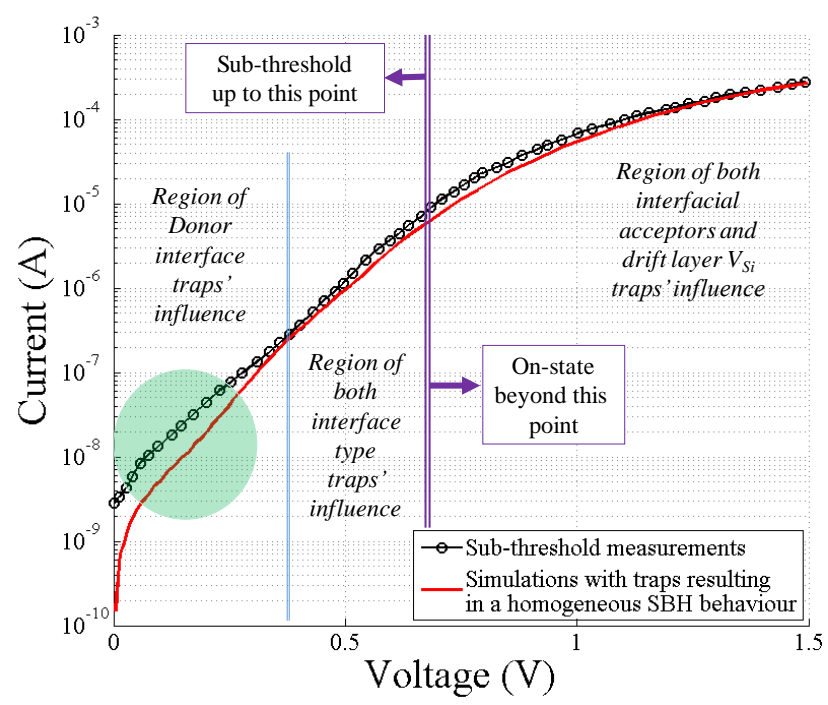

Fig. 7 The incorporation of both the bulk and interface traps model A, in Table I, in the simulations result in a very good prediction of the $\log (\mathrm{I})-\mathrm{V}$ characteristics of the fabricated 3C-SiC-on-Si LSBD. The presented regions of influence are to illustrate where each one of the aforementioned trap distributions play a key role in the fitting process followed.

The simulated $\log (\mathrm{I})-\mathrm{V}$, in Fig. 7, splits upon regions of influence from the introduced traps, in Table I. These regions illustrate how the various identified trap types in the LSBD play a key role in the fitting process with the increasing forward bias. Interestingly, these traps feature uniform spatial properties along the Schottky contact. Hence, a homogeneous behaviour of the barrier height is modelled taking into account the total effect from both the interfacial and bulk traps. This homogeneous SBH approach, after the incorporation of the aforementioned traps in the TCAD model, enables for simulations that approximate with accuracy almost the entire measured data curve besides a minor part. This part of the $\log (\mathrm{I})-\mathrm{V}$, highlighted in a green cycle in Fig. 7, suggests some degree of inhomogeneity of the $\mathrm{SBH}$ value across the fabricated contact.

\section{B. Modelling the Inhomogeneous features of the SBH with additional distribution of states at the Schottky interface}

The sub-threshold analysis in the previous sub-section indicated that for early forward bias conditions a lateral nonuniformity of the barrier height exists. Such inhomogeneous features are likely due to the large Schottky contacts on the 3C-SiC-on-Si surface [21].

The green cycled part, in Fig. 7, implies the contribution of additional states at the interface whose presence creates the conditions for more majority carriers to cross the barrier. These added states should feature a spatial distribution in a limited area of the Schottky contact active area. Thereafter, the joint effect from both the traps' model A, in Table I, and the added states will determine a weak region at the Schottky interface prone in conducting larger current for small forward bias values. Within this weak region, the $\mathrm{SBH}$ can be considered having a smaller value compared to the rest of the contact, essentially modelling an overall non-uniform behavior. The properties of these additional interfacial traps were determined with simulations and are presented in Table II as traps' model B. 
Table II. Additional identified interface traps in the fabricated $\mathrm{Pt} / 3 \mathrm{C}$ $\mathrm{SiC}$-on-Si resulting in a inhomogeneous Schottky contact.

\begin{tabular}{|c|c|c|c|c|}
\hline \multicolumn{2}{|c|}{ Trap description } & \multirow{2}{*}{$\begin{array}{c}\text { Type / } \\
\text { Concentration }\end{array}$} & \multicolumn{2}{c|}{ Distributions } \\
\cline { 3 - 5 } & & & Energetic & Spatial \\
\hline & Superimposed & & & Uniform \\
along \\
Schottky
\end{tabular}

The traps' model B consists of a donor-like distribution of interfacial states. All the energy levels of these states are above the $\left.E_{F}^{e f f}\right|_{I T}$ for zero-bias conditions. In consequence, they are considered ionized and, thus, highly likely to be occupied with holes. While occupied, they hold a positive charge. The limited spatial distribution of the traps' model B, as described in Table II, indicates a region on the Schottky contact which experiences an enhanced effect induced by the added concentrations of the charged donor-like states of both traps' model A and B. The accumulated positive charge at this region of the interface enhances the phenomena described in the previous sub-section; the lowering of the barrier becomes more intense allowing for more electrons to cross the barrier for the same bias and temperature conditions. Further, the induced band bending is stronger increasing the FE.

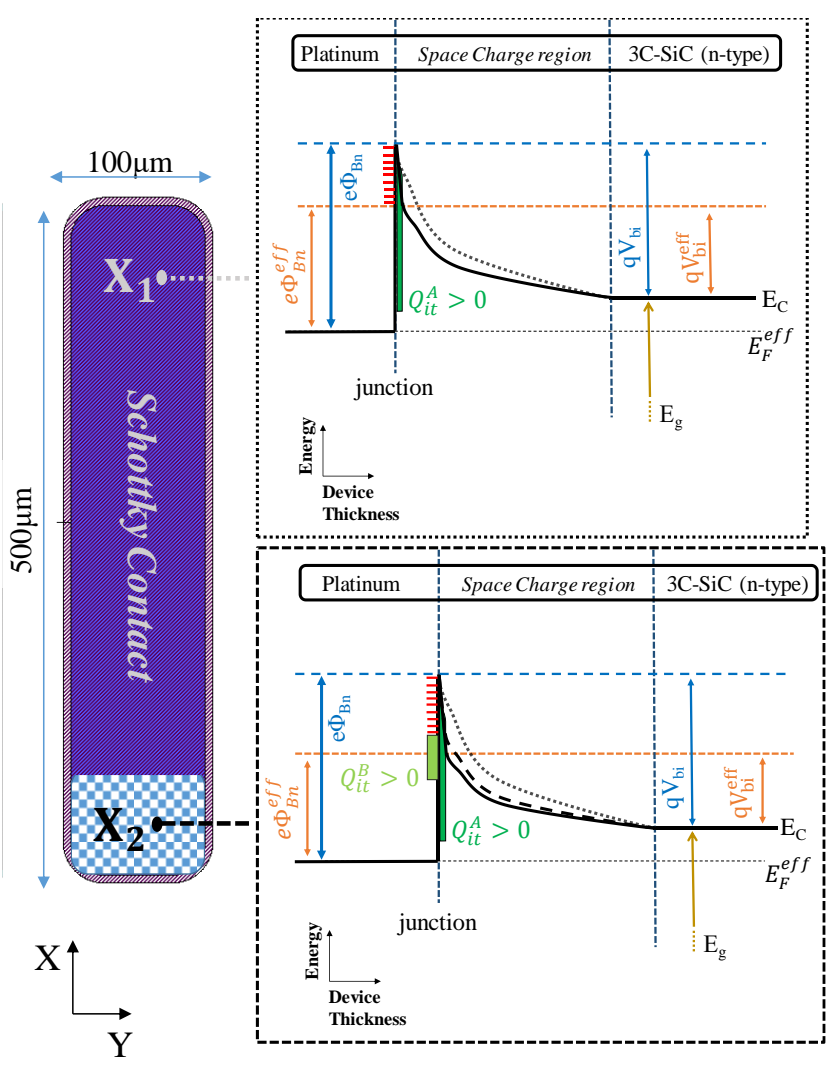

Fig. 8 The effect of the identified traps in modelling the inhomogeneous behaviour of the fabricated $3 \mathrm{C}-\mathrm{SiC}$-on-Si Schottky contacts. The X1 point is indicative of a location on the Schottky active area where the interfacial traps' model A is uniformly applied. The X2 point belongs to a portion of the Schottky active area in which the identified interfacial traps' model A and B spatially overlap. The additive effect of the traps within this portion of the contact forms a region of lower SBH value.

Fig. 8 depicts how the identified interfacial traps, in Table I and Table II, are able to model a non-uniform SBH along the Schottky contact by featuring different spatial distributions.
The X2 point belongs to the assumed weak region where the effect from both traps' model A and B applies. On the other hand, the X1 point is representative of any other point on the contact which experiences the effect from the traps' model A only. The dotted $E_{C}$ line in Fig. 8 corresponds to the initial band bending due to the space charge region formation in thermal equilibrium. This is identical to the trap-free case illustrated at the band diagram in Fig. 3. The solid $E_{C}$ line indicates the current band bending induced at each point due to the overall effect of the identified traps in Table I and Table II. Finally, the dashed $E_{C}$ line in the inset band diagram at point X2 corresponds to the initial effect from the traps' model A, before the additional traps' model B results in the final solid line realizing the inhomogeneity of the SBH.

The energy levels of the donor-like states in traps' model B expand up to the lowest level of the acceptor-like states energetic distribution in model A. This also limits their contribution on a small range of forward bias values. With the increased forward bias, the $\left.E_{F}^{e f f}\right|_{I T}$ will move upwards decreasing the number of the donor-like states that are likely to be occupied by a hole. When the forward bias reaches a sufficient value for the $\left.E_{F}^{e f f}\right|_{I T}$ to ionize the acceptor-like states, specified in the traps' model A, then the effect of the traps' model B will become negligible. Thereafter, the simulated Schottky contact will demonstrate a uniform behaviour of the SBH, as the result of the traps' model A effect only.

The developed TCAD model, which incorporates all the trap distributions in Table I and Table II, is able to accurately describe the electrical performance of the fabricated LSBD. The final excellent match achieved between measurements and simulations, in Fig. 9, adds great confidence to the validity of the combined effect from the involved carrier transport mechanisms and the identified traps. The high prediction ability of the developed models underpins the competence to design improved power device structures utilizing 3C-SiC-onSi.

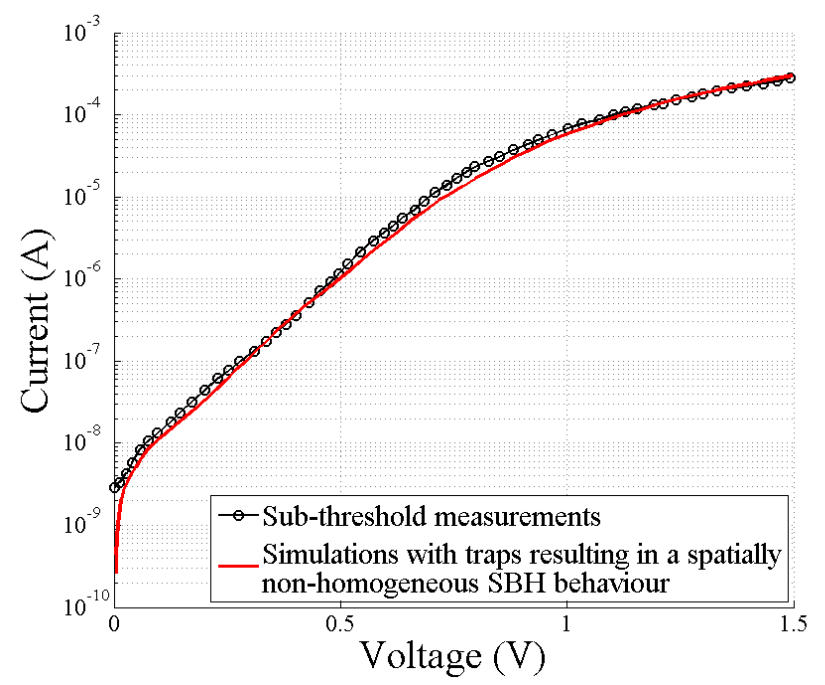

Fig. 9 The proposed TCAD model is able to accurately replicate the electrical performance of the fabricated $\mathrm{Pt} / 3 \mathrm{C}-\mathrm{SiC}$-on-Si lateral Schottky contacts by physically linking the SBH behaviour, including any observed inhomogeneous features, to the effect induced by the identified traps.

\section{CONCLUSIONS}

In this paper the fabrication of lateral 3C-SiC-on-Si SBDs is reported in an effort to investigate the origins of the leaky 
behaviour in such devices. In this context, the role of traps is highlighted as the source of the observed large leakage current, which otherwise cannot be explained by the native WBG properties of the $\mathrm{SiC}$ semiconductor.

An advanced TCAD model is proposed to link the significant degree of non-ideality with the existing traps by engaging complex trapping/de-trapping phenomena. Initially, bulk traps of acceptor type have been identified in the 3C-SiCon-Si through simulations, with activation energy below the effective Fermi level. These deep acceptor levels resemble the intrinsic $V_{S i}$ defects, introduced in 3C-SiC-on-Si due to the out-diffusion of $\mathrm{Si}$ atoms to form the $\mathrm{SiC}$ layer. Subsequently, it was established that the shape of the sub-threshold region of the $\log (\mathrm{I})-\mathrm{V}$ from the characterized LSBD is mainly affected by Schottky interfacial states. The utilization of band diagrams, in respect to semiconductor physics, has been deployed to shed light on the effect of these states and, thereafter, their energetic distribution properties where determined through extensive simulations. In particular, an interfacial traps' profile was defined, of both donor and acceptor-like states, featuring a spatially uniform distribution over the Schottky active area.

The suggested method further enabled for the SBH inhomogeneity interpretation. It was found that a distinct distribution of donor-like states spatially limited in a small portion of the Schottky active area also exists. In turn, this portion of the Schottky contact experiences an additive effect from both the uniform interfacial traps' profile and the distinct donor-like distribution emulating a lower SBH region. Moreover, the simulations revealed that the additional donorlike distribution of states should be energetically expanding in a narrower band of energies at the Schottky interface. Consequently, this results in an inhomogeneous overall behaviour of the Schottky contact with forward bias.

After the inclusion of all the identified traps, both bulk and interfacial, the TCAD model is able to predict the characterized $\log (\mathrm{I})-\mathrm{V}$ of the fabricated $3 \mathrm{C}$-SiC-on-Si LSBD with excellent accuracy. The suggested model is not limited to 3C-SiC-on-Si, rather it can be applied for any Metal / Semiconductor junction to elucidate the effect from the inevitable presence of traps on the SBH and the electrical performance of the device. The contribution of this work could potentially assist in optimizing the fabrication strategies leading to a reduction of these traps. In turn, this can be of immense value towards monolithic integration and an effective co-existence of 3C-SiC-on-Si and Si devices

\section{REFERENCES}

[1] J. Millan, P. Godignon, X. Perpina, A. Perez-Tomas, and J. Rebollo, "A Survey of Wide Bandgap Power Semiconductor Devices," IEEE Trans. Power Electron., vol. 29, no. 5, pp. 2155-2163, 2014. doi:10.1109/TPEL.2013.2268900

[2] A. Elasser and T. P. Chow, "Silicon carbide benefits and advantages for power electronics circuits and systems," Proc. IEEE, vol. 90, no. 6, pp. 969-986, 2002. doi:10.1109/JPROC.2002.1021562

[3] T. P. Chow, "High-voltage $\mathrm{SiC}$ and $\mathrm{GaN}$ power devices," Microelectron. Eng., vol. 83, no. 1, pp. 112-122, 2006 doi:10.1016/j.mee.2005.10.057

[4] J. L. Hudgins, G. S. Simin, E. Santi, and M. A. Khan, "An assessment of wide bandgap semiconductors for power devices," IEEE Trans. Power Electron., vol. 18, no. 3, pp. 907-914, 2003. doi:10.1109/TPEL.2003.810840

[5] M. Kanechika, T. Uesugi, and T. Kachi, "Advanced $\mathrm{SiC}$ and GaN power electronics for automotive systems," in Technical Digest -
International Electron Devices Meeting, IEDM, 2010. doi:10.1109/IEDM.2010.5703356, pp. 324-327

[6] G. Ferro, "3C-SiC Heteroepitaxial Growth on Silicon: The Quest for Holy Grail," Crit. Rev. Solid State Mater. Sci., vol. 40, no. 1, pp. 5676, 2015. doi:10.1080/10408436.2014.940440

[7] F. La Via, A. Severino, R. Anzalone, C. Bongiorno, G. Litrico, M. Mauceri, M. Schoeler, P. Schuh, and P. Wellmann, "From thin film to bulk 3C-SiC growth: Understanding the mechanism of defects reduction," Mater. Sci. Semicond. Process., vol. 78, pp. 57-68, 2018. doi:10.1016/j.mssp.2017.12.012

[8] H. Nagasawa, K. Yagi, T. Kawahara, and N. Hatta, "Properties of FreeStanding 3C-SiC Monocrystals grown on Undulant- Si ( 001 ) Substrate," vol. $\quad 436, \quad$ pp. $\quad 3-8, \quad 2003$. doi:10.4028/www.scientific.net/MSF.433-436.3

[9] K. Sasaki, E. Sakuma, S. Misawa, S. Yoshida, and S. Gonda, "Hightemperature electrical properties of $3 \mathrm{C}-\mathrm{SiC}$ epitaxial layers grown by chemical vapor deposition," Appl. Phys. Lett., vol. 45, no. 1, pp. 7273, 1984. doi: $10.1063 / 1.94973$

[10] A. A. Lebedev, S. P. Lebedev, V. Y. Davydov, S. N. Novikov, and Y. N. Makarov, "Growth and investigation SiC based heterostructures," in 15th Biennial Baltic Electronics Conference (BEC), 2016. doi:10.1109/BEC.2016.7743717, pp. 5-6

[11] O. Bouketir, "Advances and Challenges in WBG Devices and their Applications in Power Conversion and Conditioning," in Proceedings of the International Conference on Recent Advances in Electrical Systems, 2016, pp. 34-40

[12] S. Roy, C. Jacob, and S. Basu, "Current transport properties of Pd/3C$\mathrm{SiC}$ Schottky junctions with planar and vertical structures," Solid State Sci., vol. 6, no. 4, pp. 377-382, 2004. doi:10.1016/j.solidstatesciences.2004.01.003

[13] A. Severino, G. D’Arrigo, C. Bongiorno, S. Scalese, F. La Via, and G. Foti, "Thin crystalline $3 \mathrm{C}-\mathrm{SiC}$ layer growth through carbonization of differently oriented Si substrates," J. Appl. Phys., vol. 102, no. 2, 2007. doi:10.1063/1.2756620

[14] A. Stefanskyi, Ł. Starzak, and A. Napieralski, "Silicon Carbide Power Electronics for Electric Vehicles," 2015 Tenth Int. Conf. Ecol. Veh. Renew. Energies, pp. 1-9, 2015. doi:10.1109/EVER.2015.7138047

[15] G. Colston, S. D. Rhead, V. A. Shah, O. J. Newell, I. P. Dolbnya, D. R. Leadley, and M. Myronov, "Mapping the strain and tilt of a suspended 3C-SiC membrane through micro X-ray diffraction," Mater. Des., vol. 103, pp. 244-248, 2016. doi:10.1016/j.matdes.2016.04.078

[16] Y. Li, Z. Zhao, L. Yu, Y. Wang, Z. Yin, Z. Li, and P. Han, "Heteroepitaxial 3C-SiC on $\mathrm{Si}\left(\begin{array}{lll}1 & 0 & 0\end{array}\right)$ with flow-modulated carbonization process conditions," J. Cryst. Growth, vol. 506, no. $\begin{array}{llll}\text { September } 2018, \quad \text { pp. } & 114-116, & 2019 .\end{array}$ doi:10.1016/j.jcrysgro.2018.09.037

[17] X. Song, J. F. Michaud, F. Cayrel, M. Zielinski, M. Portail, T. Chassagne, E. Collard, and D. Alquier, "Evidence of electrical activity of extended defects in 3C-SiC grown on Si," Appl. Phys. Lett., vol. 96, no. 14, pp. 1-4, 2010. doi:10.1063/1.3383233

[18] P. Hens, G. Wagner, A. Hölzing, R. Hock, and P. Wellmann, "Dependence of the seed layer quality on different temperature rampup conditions for 3C-SiC hetero-epitaxy on $\mathrm{Si}(100)$," Thin Solid Films, vol. 522, pp. 2-6, 2012. doi:10.1016/j.tsf.2011.10.177

[19] S. R. Nutt, D. J. Smith, H. J. Kim, and R. F. Davis, "Interface structures in beta-silicon carbide thin films," Appl. Phys. Lett., vol. 50, no. 4, pp. 203-205, 1987. doi:10.1063/1.97661

[20] W. Monch, "On the physics of metal-semiconductor interfaces," Reports Prog. Phys., vol. 53, no. 3, pp. 221-278, Mar. 1990. doi:10.1088/0034-4885/53/3/001

[21] J. Eriksson, M. H. Weng, F. Roccaforte, F. Giannazzo, S. Leone, and V. Raineri, "Toward an ideal Schottky barrier on 3C -SiC," Appl. Phys. Lett., vol. 95, no. 8, 2009. doi:10.1063/1.3211965

[22] R. T. Tung, "Electron transport at metal-semiconductor interfaces: General theory," Phys. Rev. B, vol. 45, no. 23, pp. 13509-13523, 1992

[23] J. H. Werner and H. H. Güttler, "Barrier inhomogeneities at Schottky contacts," J. Appl. Phys., vol. 69, no. 3, pp. 1522-1533, 1991. doi:10.1063/1.347243

[24] S. U. Omar, T. S. Sudarshan, T. A. Rana, H. Song, and M. V. S. Chandrashekhar, "Large barrier, highly uniform and reproducible Ni$\mathrm{Si} / 4 \mathrm{H}-\mathrm{SiC}$ forward Schottky diode characteristics: Testing the limits of Tung's model," J. Phys. D. Appl. Phys., vol. 47, no. 29, 2014. doi:10.1088/0022-3727/47/29/295102 
[25] J. L. Li, Y. Li, L. Wang, Y. Xu, F. Yan, P. Han, and X. L. Ji, "Influence of deep defects on electrical properties of $\mathrm{Ni} / 4 \mathrm{H}-\mathrm{SiC}$ Schottky diode," Chinese Phys. B, vol. 28, no. 2, 2019. doi:10.1088/16741056/28/2/027303

[26] D. Defives, O. Noblanc, C. Dua, C. Brylinski, M. Barthula, and F. Meyer, "Electrical characterization of inhomogeneous $\mathrm{Ti} / 4 \mathrm{H}-\mathrm{SiC}$ Schottky contacts," Mater. Sci. Eng. R Reports, vol. 62, pp. 395-401, 1999. doi:10.1016/S0921-5107(98)00541-8

[27] B. J. Skromme, E. Luckowski, K. Moore, M. Bhatnagar, C. E. Weitzel, T. Gehoski, and D. Ganser, "Electrical characteristics of Schottky barriers on 4H-SiC: The effects of barrier height nonuniformity," $J$ Electron. Mater., vol. 29, no. 3, pp. 376-383, 2000. doi:10.1007/s11664-000-0081-9

[28] I. Nikitina, K. Vassilevski, A. Horsfall, N. Wright, A. G. O’Neill, S. K. Ray, K. Zekentes, and C. M. Johnson, "Phase composition and electrical characteristics of nickel silicide Schottky contacts formed on 4H-SiC," Semicond. Sci. Technol., vol. 24, no. 5, 2009. doi:10.1088/0268-1242/24/5/055006

[29] X. Ma, P. Sadagopan, and T. S. Sudarshan, "Investigation on barrier inhomogeneities in 4H-SiC Schottky rectifiers," Phys. Status Solidi Appl. Mater. Sci., vol. 203, no. 3, pp. 643-650, 2006. doi:10.1002/pssa.200521017

[30] P. M. Gammon, E. Donchev, A. Pérez-Tomás, V. A. Shah, J. S. Pang, P. K. Petrov, M. R. Jennings, C. A. Fisher, P. A. Mawby, D. R. Leadley, and N. McN. Alford, "A study of temperature-related non-linearity at the metal-silicon interface," J. Appl. Phys., vol. 112, no. 11, 2012. doi:10.1063/1.4768718

[31] V. Kumar, A. S. Maan, and J. Akhtar, "Barrier height inhomogeneities induced anomaly in thermal sensitivity of Ni/4H-SiC Schottky diode temperature sensor," J. Vac. Sci. Technol. B, Nanotechnol. Microelectron. Mater. Process. Meas. Phenom., vol. 32, no. 4, p. 041203, 2014. doi:10.1116/1.4884756

[32] M. E. Aydin, N. Yildirim, and A. Türüt, "Temperature-dependent behavior of Ni/4H-nSiC Schottky contacts," J. Appl. Phys., vol. 102, no. 4, 2007. doi:10.1063/1.2769284

[33] G. S. Chung, K. S. Kim, and F. Yakuphanoglu, "Electrical characterization of $\mathrm{Au} / 3 \mathrm{C}-\mathrm{SiC} / \mathrm{n}-\mathrm{Si} / \mathrm{Al}$ Schottky junction," J. Alloys Compd., vol. 507, no. 2, pp. 508-512, 2010. doi:10.1016/j.jallcom.2010.08.004

[34] R. T. Tung, "Electron transport at metal-semiconductor interfaces: General theory," Phys. Rev. B, vol. 45, no. 23, pp. 13509-13523, 1992. doi:10.1103/PhysRevB.45.13509

[35] R. T. Tung, "Recent advances in Schottky barrier concepts," Mater. Sci. Eng. $R$ Reports, vol. 35, no. 1-3, pp. 1-138, 2001. doi:10.1016/S0927-796X(01)00037-7

[36] J. P. Sullivan, R. T. Tung, M. R. Pinto, and W. R. Graham, "Electron transport of inhomogeneous Schottky barriers : A numerical study," $J$. Appl. Phys., vol. 70, no. 12, pp. 7403-7424, 1991. doi:10.1063/1.349737

[37] H.-J. Im, Y. Ding, J. P. Pelz, and W. J. Choyke, "Nanometer-scale test of the Tung model of Schottky-barrier height inhomogeneity," Phys. Rev. B, vol. 64, no. 7, p. 075310, 2001. doi:10.1103/PhysRevB.64.075310
[38] F. La Via, M. Camarda, and A. La Magna, "Mechanisms of growth and defect properties of epitaxial SiC," Appl. Phys. Rev., vol. 1, no. 3, 2014. doi:10.1063/1.4890974

[39] Y. Yamamoto, S. Harada, K. Seki, A. Horio, T. Mitsuhashi, D. Koike, M. Tagawa, and T. Ujihara, "Low-dislocation-density 4H-SiC crystal growth utilizing dislocation conversion during solution method," Appl. Phys. Express, vol. 7, no. 6, 2014. doi:10.7567/APEX.7.065501

[40] M. Benamara, M. Anani, B. Akkal, and Z. Benamara, "Ni/SiC-6H Schottky Barrier Diode interfacial states characterization related to temperature," J. Alloys Compd., vol. 603, pp. 197-201, 2014. doi:10.1016/j.jallcom.2014.02.177

[41] K. Y. Lee and Y. H. Huang, "An investigation on barrier inhomogeneities of $4 \mathrm{H}-\mathrm{SiC}$ Schottky barrier diodes induced by surface morphology and traps," IEEE Trans. Electron Devices, vol. 59, no. 3, pp. 694-699, 2012. doi:10.1109/TED.2011.2181391

[42] T. Katsuno, Y. Watanabe, H. Fujiwara, M. Konishi, H. Naruoka, J. Morimoto, T. Morino, and T. Endo, "Analysis of surface morphology at leakage current sources of 4H-SiC Schottky barrier diodes," Appl. Phys. Lett., vol. 98, no. 22, pp. 2-5, 2011. doi:10.1063/1.3597413

[43] K. Y. Lee and M. A. Capano, "The correlation of surface defects and reverse breakdown of $4 \mathrm{H}-\mathrm{SiC}$ Schottky barrier diodes," J. Electron. Mater., vol. 36, no. 4, pp. 272-276, 2007. doi:10.1007/s11664-0060075-3

[44] A. Arvanitopoulos, N. Lophitis, K. N. Gyftakis, S. Perkins, and M. Antoniou, "Validated physical models and parameters of bulk 3C-SiC aiming for credible technology computer aided design (TCAD) simulation," Semicond. Sci. Technol., vol. 32, no. 10, p. 104009, 2017. doi:10.1088/1361-6641/aa856b

[45] N. Lophitis, A. Arvanitopoulos, S. Perkins, and M. Antoniou, "TCAD Device Modelling and Simulation of Wide Bandgap Power Semiconductors," in Disruptive Wide Bandgap Semiconductors, Related Technologies, and Their Applications, Y. K. Sharma, Ed. Rijeka: InTech, 2018

[46] Keysight, "Keysight B1505A Power Device Analyzer/Curve Tracer." 2011

[47] T. J. Drummond, "Work Functions of the Transition Metals and Metal Silicides," Albuquerque, 1999

[48] L. Wenchang, Z. Kaiming, and X. Xide, "An electronic structure study of single native defects in beta -SiC," J. Phys. Condens. Matter, vol. 5, no. 7, pp. 891-898, Feb. 1993. doi:10.1088/0953-8984/5/7/016

[49] M. Mandurrino, G. Verzellesi, M. Goano, M. E. Vallone, F. Bertazzi, G. Ghione, M. Meneghini, G. Meneghesso, and E. Zanoni, "Trapassisted tunneling in InGaN/GaN LEDs: Experiments and physicsbased simulation," Proc. Int. Conf. Numer. Simul. Optoelectron. Devices, NUSOD, 2014. doi:10.1109/NUSOD.2014.6935332

[50] B. Asllani, M. Berthou, D. Tournier, P. Brosselard, and P. Godignon, "Modeling of Inhomogeneous 4H-SiC Schottky and JBS Diodes in a Wide Temperature Range," Mater. Sci. Forum, vol. 858, pp. 741-744, 2016 